\title{
Magnetic Response of Mn(III)F(salen) at Low Temperatures
}

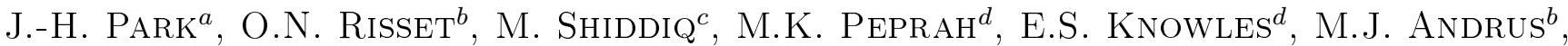 \\ C.C. Beedle ${ }^{a}$, G. Ehlers ${ }^{e}$, A. Podlesnyak ${ }^{e}$, E. ČižMár ${ }^{f}$, S.E. NAgler ${ }^{e}$, S. Hill $^{c}$, \\ D.R. TALHAM ${ }^{b}$, M.W. MEISEL ${ }^{d, f, *}$ \\ ${ }^{a}$ National High Magnetic Field Laboratory (NHMFL), Florida State Univ., Tallahassee, FL 32310-3706, USA \\ ${ }^{b}$ Department of Chemistry, University of Florida, Gainesville, FL 32611-7200, USA \\ ${ }^{c}$ Department of Physics and NHMFL, Florida State University, Tallahassee, FL 32310-3706, USA \\ ${ }^{d}$ Department of Physics and NHMFL, University of Florida, Gainesville, FL 32611-8440, USA \\ ${ }^{e}$ Quantum Condensed Matter Division, Oak Ridge National Laboratory, Oak Ridge, TN 37831-6477, USA \\ ${ }^{f}$ Institute of Physics, Faculty of Science, P.J. Šafárik University, Park Angelinum 9, 04154 Košice, Slovakia
}

The low temperature magnetic response of $\mathrm{Mn}(\mathrm{III}) \mathrm{F}$ (salen), salen $=\mathrm{H}_{14} \mathrm{C}_{16} \mathrm{~N}_{2} \mathrm{O}_{2}$, an $S=2$ linear-chain system, has been studied. Using a single crystal with the field applied perpendicular to the chain direction, torque magnetometry, down to $20 \mathrm{mK}$ and up to $18 \mathrm{~T}$, revealed a feature at $3.8 \mathrm{~T}$ when $T \leq 400 \mathrm{mK}$. ESR $(\approx 200 \mathrm{GHz})$ studies, using single crystals at $4 \mathrm{~K}$ and in $5 \mathrm{~T}$, have not detected any signal. In $10 \mathrm{mT}$, the temperature dependence of the susceptibility of powder-like samples can be reasonably fit when $J / k_{B}=50 \mathrm{~K}$ and $g=2$. In addition, these data are unchanged for $P \leq 1.0 \mathrm{GPa}$. Using a randomly-oriented, powder-like, deuterated (12 of $14 \mathrm{H}$ replaced by D) sample of $2.2 \mathrm{~g}$ at $270 \mathrm{mK}$, neutron scattering data, acquired with the Cold Neutron Chopper Spectrometer at the Spallation Neutron Source, show several well defined excitations that may be from the zero-field energy levels of antiferromagnetic $S=2$ spins with $g=2, J / k_{B}=50 \mathrm{~K}, D / k_{B}=2.8 \mathrm{~K}$, and $E / k_{B}=0.5 \mathrm{~K}$.

DOI: 10.12693/APhysPolA.126.228

PACS: 75.50.Ee, 78.70.Nx, 76.30.-v, 75.10.Jm

\section{Introduction}

Linear-chain, integer-spin antiferromagnets, commonly known as Haldane systems, have a storied history in the field of quantum spins, with $S=1$ materials receiving the most attention [1]. The $S=2$ systems have received less consideration for several reasons, including a smaller Haldane gap, since $\Delta_{S=1}=0.41 \mathrm{~J}$ and $\Delta_{S=2}=0.09 J$, where $J$ is the nearest-neighbor exchange energy [2]. Due to a report suggesting $\mathrm{Mn}(\mathrm{III}) \mathrm{F}$ (salen) is an $S=2$ Haldane system with $J / k_{B}=50 \mathrm{~K}$ and no evidence of long-range order down to $2 \mathrm{~K}$ [3], we have studied the magnetic response of single-crystals at low temperatures and in high magnetic fields.

\section{Experimental results}

Using a single crystal with the field applied perpendicular to the chain direction, torque magnetometry, down to $20 \mathrm{mK}$ and up to $18 \mathrm{~T}$, revealed a reproducible feature in the response of a torque magnetometer [4], Fig. 1. In addition, neutron scattering data acquired with a randomly-oriented, powder-like, deuterated (12 of $14 \mathrm{H}$ replaced by D) sample of $2.2 \mathrm{~g}$ at $270 \mathrm{mK}$ and obtained with the CNCS (Cold Neutron Chopper Spectrometer) at the SNS (Spallation Neutron Source) show several well defined excitations, Fig. 2. Finally, EPR studies

*corresponding author; e-mail: meisel@phys.ufl.edu near $200 \mathrm{GHz}$ have not identified a response from single crystals at $4 \mathrm{~K}$ and in $5 \mathrm{~T}$, and the low-field (10 mT), high-temperature (up to $300 \mathrm{~K}$ ) magnetic susceptibility, $\chi(T)$, of randomly oriented microcrystals is unchanged for $P<1.0 \mathrm{GPa}$.

\section{Discussion and summary}

Our $\chi(T)$ results can be simulated with $J / k_{B} \approx$ $50 \mathrm{~K}$ [5], which is close to the value reported by Birk et al. [3]. Following the analysis used with $\mathrm{Mn}(\mathrm{III}) \mathrm{Cl}_{3}$ (bipy), an $S=2$ Haldane system [6, 7], our magnetometry results indicate the possible presence of a critical field of $3.8 \mathrm{~T}$. Assuming $\Delta_{S=2}=0.09 \mathrm{~J}$ and $g=2$, this field yields $J / k_{B}=53 \mathrm{~K}$. Recently, highfield EPR work [8] was used to determine the single-ion anisotropies $D$ and $E$ in $\mathrm{Mn}(\mathrm{III}) \mathrm{Cl}_{3}$ (bipy), where two zero-field modes with energies $\propto J(D \pm E)$ were detected. If $\mathrm{MnF}$ (III) $\mathrm{F}$ (salen) behaves in a similar fashion and taking $J / k_{B}=50 \mathrm{~K}$ from our $\chi(T)$ data, the features at $4.5 \mathrm{meV}$ and $5.4 \mathrm{meV}$ in Fig. 2 suggest $D / k_{B}=2.8 \mathrm{~K}$ and $E / k_{B}=0.5 \mathrm{~K}$ for $g=2$.

It is too early to determine if $\mathrm{Mn}$ (III)F(salen) is an "even" Haldane system or an XY material $[9,10]$. The local environments of the Mn are complicated, with three distinct Mn per unit cell in a $-1-2-3-3-2-1-$ chain motif where three different bridging $\mathrm{Mn}-\mathrm{F}-\mathrm{Mn}$ angles are present [3]. Our characterization of this interesting material continues. 


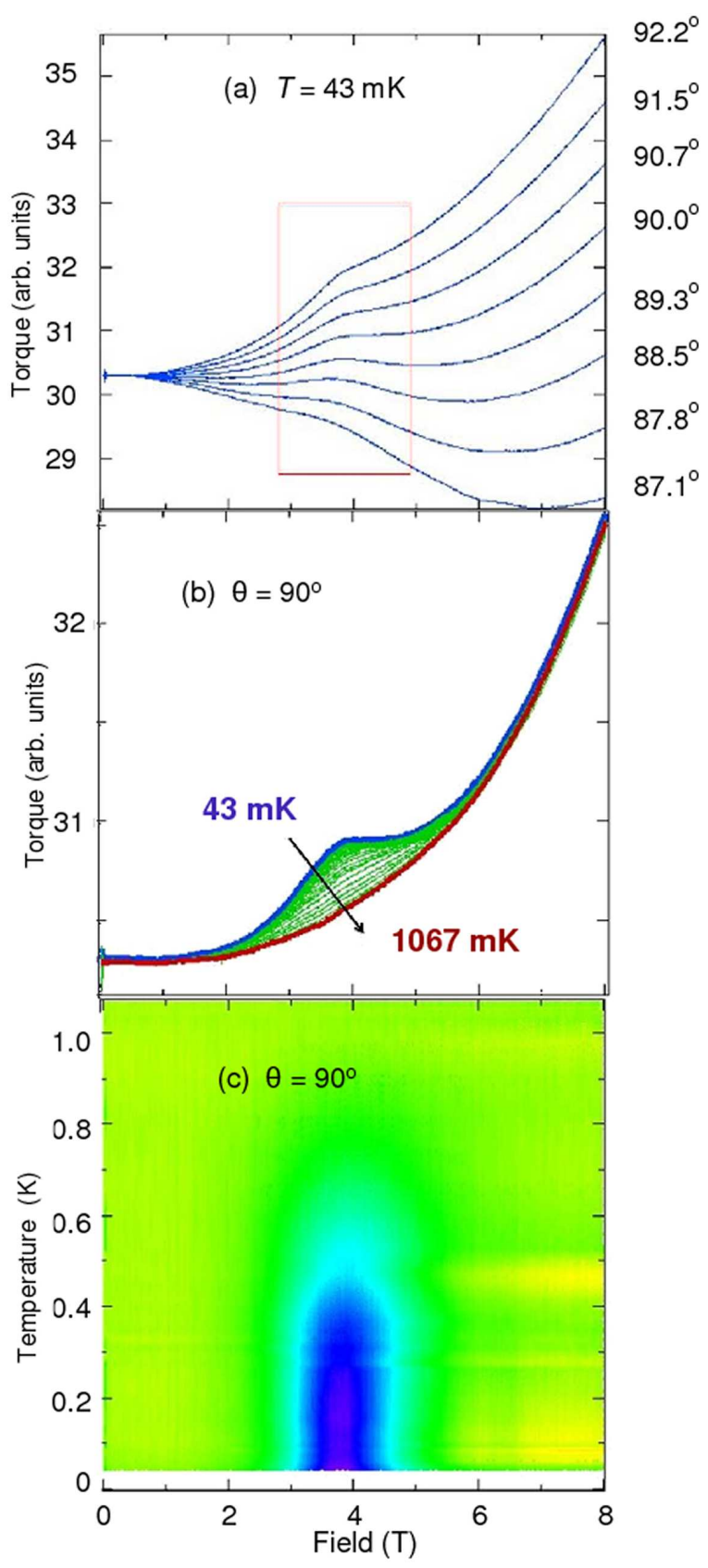

Fig. 1. The temperature and magnetic field responses of the torque magnetometer, (a) for various orientations of the field with the chain axis at $43 \mathrm{mK}$, where the values of the angle $\theta$ are given and $\theta=90^{\circ}$ is the angle between the magnetic field and the magnetic easy-axis, where the detected torque was null below $2 \mathrm{~T}$, and (b) with fixed orientation and different temperatures. Panel (c) shows the weak (green) to strong (blue to pink) response as a color gradient, indicating the presence of a critical field, $H_{C 1}=3.8 \mathrm{~T}$.

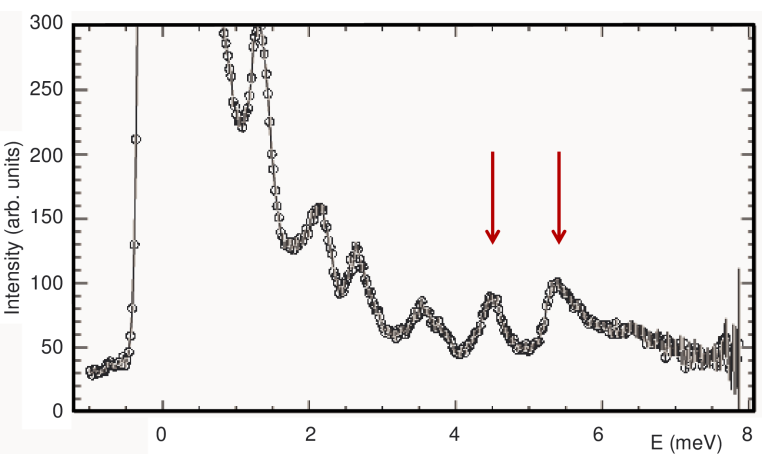

Fig. 2. The intensity versus energy obtained at $270 \mathrm{mK}$, for $0.9 \AA^{-1} \leq \mathrm{Q} \leq 1.2 \AA^{-1}$ at an incident energy of $10 \mathrm{meV}$. The red arrows indicate the modes used in the analysis, see text.

\section{Acknowledgments}

Discussions with M. Hagiwara, G. E. Granroth, T. Hong, S. E. Brown, and J. Strečka have been very helpful. Work supported by NSF via DMR-1005581 (DRT), DMR-0804408 (SH), DMR-1202033 (MWM), and DMR1157490 (NHMFL); by the Slovak Agency for Support of Research and Development APVV-0132-11; by the Fulbright Commission of the Slovak Republic (MWM). Research at Oak Ridge National Laboratory's SNS was supported by the Scientific User Facilities Division, Office of Basic Energy Sciences, U.S. Department of Energy.

\section{References}

[1] M. Yamashita, T. Ishii, H. Matsuzaka, Coord. Chem Rev. 198, 347 (2000).

[2] S. Todo, K. Kato, Phys. Rev. Lett. 87, 047203 (2001).

[3] T. Birk, K.S. Pedersen, S. Piligkos, C.Aa. Thuesen, H. Weihe, J. Bendix, Inorg. Chem. 50, 5312 (2011).

[4] E. Ohmichi, T. Osada, Rev. Sci. Instrum. 73, 3022 (2002).

[5] J.M. Lw, H. Benner, R.K. Kremer, J. Phys. Condens. Matter 25, 065601 (2013).

[6] G.E. Granroth, M.W. Meisel, M. Chaparala, Th. Jolicœur, B.H. Ward, D.R. Talham, Phys. Rev. Lett. 77, 1616 (1996).

[7] G.E. Granroth, S.E. Nagler, R. Coldea, R.S. Eccleston, B.H. Ward, D.R. Talham, M.W. Meisel, Applied Physics A 74, S868 (2002).

[8] M. Hagiwara, M. Ikeda, Y. Idutsu, S. Kimura, Z. Honda, J. Korean Phys. Soc. 62, 2046 (2013).

[9] S.C. Furuya, M. Oshikawa, I. Affleck, Phys. Rev. B 83, 224417 (2011).

[10] J.A. Kjäll, M.P. Zaletel, R.S.K. Mong, J.H. Bardarson, F. Pollmann, Phys. Rev. B 87, 235106 (2013). 